\title{
ESTIMATING EXPERIMENTALLY \\ ACCURACY OBTAINED SYSTEM ZIP LOAD MODELS
}

\author{
Aleksey V. Pankratov ${ }^{*}$, Igor E. Kopylov ${ }^{1}$, Alexander S. Tavlintsev ${ }^{2}$, and \\ Mikhail A. Kondrashov ${ }^{1}$, and $L i$ Wang $^{3}$ \\ ${ }^{1}$ Tomsk Polytechnic University, 634050 Tomsk, Russia \\ ${ }^{2}$ Ural Federal University, 620002 Ekaterinburg, Russia \\ ${ }^{3}$ National Cheng Kung University, Tainan City 701, Taiwan
}

\begin{abstract}
Experimentally obtained ZIP load models are used for the power system analysis. Estimating accuracy of such models is presented in this paper. The dependence of ZIP load model error on the range in which voltage changes during the experiment, the number of measured values of voltage and power, standard deviation of the load change random process have been obtained using the computing experiment. The results could be used to estimate the accuracy of ZIP load models or to design the experiment to determine ZIP load models with prescribed accuracy.
\end{abstract}

\section{Introduction}

Load models are necessary for power system study. The impact of the load model on voltage stability is demonstrated in [1,2]. Different load model types and international industry practice on load modeling are described in [3]. Generally all existing load models can be classified in two groups: static load models [1, 4] and dynamic load models [5, 6]. Static load model in the form of parabolic curve contains three components: constant resistance, constant current and constant power. This model was called as ZIP model. ZIP load model is widely used [3].

Load rate and structure vary over time [4,6]. It leads to the necessity of periodic model parameters reassessment. For large load centers the static load models are determined by means of special experimental researches. Despite the large number of studies, devoted to the measurement based load model identification [1, 4, 6, 7], the problem of obtained models accuracy remains actual till the present day.

The research objective is to calculate the accuracy of ZIP load models obtained experimentally depending on the parameters of the experiment. The parameters of the experiment influencing the accuracy of ZIP load models are the following: the range in which voltage changes during the experiment $(\Delta u \%)$; the number of measured values of voltage and power $(N)$; a standard deviation of the load change random process $\left(\sigma_{p} \%\right)$.

\footnotetext{
*Corresponding author: pank@tpu.ru
} 


\section{Research methods}

The difficulty of estimating the accuracy of the ZIP load models lies in the fact that the standard which the result obtained in the experiment could be compared with is lacking. So the authors have decided to use a computing experiment instead of a full-scale one.

The initial reference static load model was defined in relative units by the $2^{\text {nd }}$ degree polynomial (1) with known coefficients $a_{0}^{\prime}, a_{1}^{\prime}, a_{2}^{\prime}$ :

$$
P_{\text {ORIG }}(U)=a_{0}^{\prime}+a_{1}^{\prime} \cdot U+a_{2}^{\prime} \cdot U^{2}
$$

Arrays of voltage values $U_{(i)}$ and real power $P_{(i)}$, where $i=1,2, \ldots, N$ is the index of reading, were determined in the computer experiment. The values $U_{(i)}$ have been obtained in relative units on the assumption that voltage increases regularly during the experiment within the limits $\pm \Delta \mathrm{u}_{\%} / 2$ from the rated voltage according to (2):

$$
U_{(i)}=1-\frac{\Delta u_{0}}{2 \cdot 100 \%}+i \cdot \frac{\Delta u_{\%}}{N \cdot 100 \%} .
$$

The influence of the random error of measurements and random fluctuations of the load power on the accuracy of the obtained static load models may be generalized by taking into account one random normally distributed value with a standard deviation $\sigma_{p \%}$ :

$$
P_{(i)}=\left(a_{0}^{\prime}+a_{1}^{\prime} \cdot U_{(i)}+a_{2}^{\prime} \cdot U_{(i)}^{2}\right) \cdot \operatorname{error}\left(\sigma_{p \%}\right)
$$

where $\operatorname{error}\left(\sigma_{p \%}\right)$ is the random normally distributed value with the mean value of 1 .

Approximation initial data $U_{(i)}$ and $P_{(i)}$ by $2^{\text {nd }}$ degree polynomial using ordinary least squares method gives the experimental static load model with new coefficients $a_{0}, a_{1}, a_{2}$ :

$$
P_{T E S T}(U)=a_{0}+a_{1} \cdot U+a_{2} \cdot U^{2}
$$

The results of one experiment are shown in fig. 1.

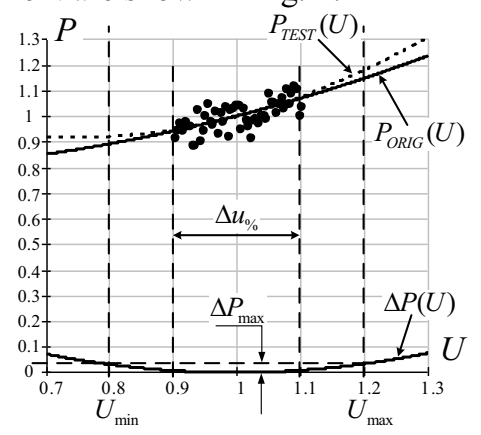

Fig. 1. Comparison of reference and experimental static load models.

The voltage was varied within the $\Delta u \%$ range during the experiment. A comparison of the reference static load model $P_{\text {ORIG }}(U)$ and the experimental static load model $P_{T E S T}(U)$ is performed within the predetermined range $\left(U_{\min } \div U_{\max }\right)$ that was chosen on the basis of the voltage variation deviation under normal and post-emergency conditions $(0.8 / 1.2$ from the rated voltage). The maximum deviation of the models $\Delta P(U)=\left|P_{O R I G}(U)-P_{T E S T}(U)\right|$ within 
the predetermined range $\left(U_{\min } \div U_{\max }\right)$ of $\Delta P_{\max }$ was used to obtain the numerical value of the error of the static load models.

The random values $P_{(i)}$ were used as the initial data so the obtained result $\Delta P_{\max }$ is also random with the same values $\Delta u \%, N$, and $\sigma_{p} \%$. The results of the set of experiments are presented in fig. 2.

The values $\Delta P_{\max }$ obtained in the set of $S=1000$ computing experiment are shown by dots in fig. 2a. The probability-distribution function of the values $\Delta P_{\max }$ is shown in fig. $2 \mathrm{~b}$. The value of the quantile $\Delta_{\max }$ was taken as the final value of the static load model's error. This value will not exceed the probability of 0.95 .

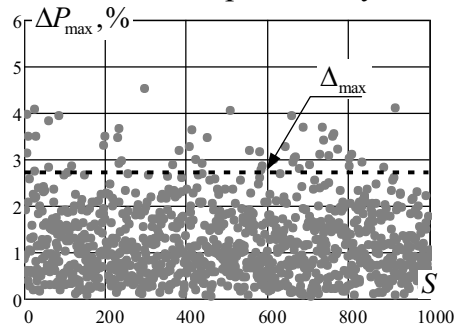

a).

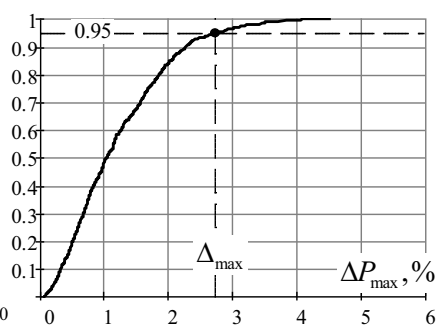

b).

Fig. 2. a) set of computing experiments; b) probability-distribution function.

\section{Obtained results}

The described sets of experiments were carried out for the different values $\Delta u \%, N$ and $\sigma_{p} \%$. The dependences of the static load model's error $\Delta_{\max }$ on the number of measurements $N$ for various values $\Delta u \%$, with $\sigma_{p \%}=1 \%$ are shown in fig. 3 .
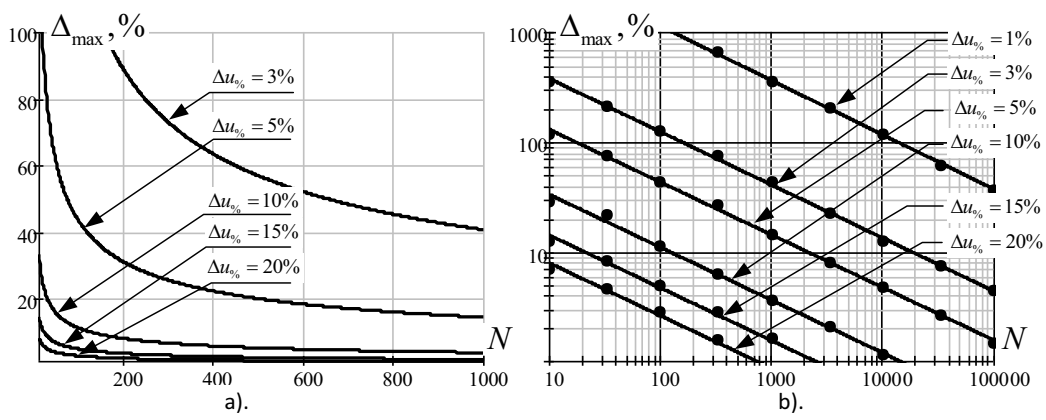

Fig. 3. Values of the static load model's error with $\sigma_{p} \%=1 \%$ : a)linear scales; b) logarithmic scales.

These dependences are linear on the logarithmic scales (fig. 3b), so they can be approximated by the linear functions. The same linear functions can be obtained for $\Delta_{\max }(\Delta u \%)$ and $\Delta_{\max }\left(\sigma_{p} \%\right)$ dependences. Finally all results are generalized by equation

$$
\Delta_{\max }=118 \cdot \frac{\sigma_{p \%}}{(\Delta u \%)^{2} \cdot \sqrt{N}} \cdot 100 \%
$$

Thus, given the known values of the range of the voltage variation $\Delta u \%$, the number of measured values $N$ and the standard deviation of load power $\sigma_{p} \%$, the resulting error of the static load model will not exceed the value $\Delta_{\max }$ calculated from (5), with the probability of 0.95. For example, with $\sigma_{p \%}=1 \%$ and $\Delta u \%=20 \%$, and $N=10^{3}$, the error of the static load 
models will not exceed the value $\Delta_{\max }=0.93 \%$, calculated from (5), with the probability of 0.95. If $\sigma_{p} \%=10 \%, \Delta u \%=5 \%, N=10^{3}$, then $\Delta_{\max }=149 \%$.

The inverse problem (design of the experiment for determining the static load models with the prescribed accuracy) could be solved using the obtained results. For example, if the accuracy of the static load model does not exceed $10 \%$ and the tolerance range of the voltage variation is $\Delta u \%=15 \%$, then with $\sigma_{p}=10 \%$, solving (5) for $N$, we will obtain

$$
N=\left(\frac{118 \cdot \sigma_{p \%} \cdot 100 \%}{(\Delta u \%)^{2} \cdot \Delta_{\max }}\right)^{2}=\left(\frac{118 \cdot 10 \cdot 100}{15^{2} \cdot 10}\right)^{2}=2750 .
$$

Therefore, under these conditions, for the measurements to obtain the required accuracy it is necessary that $N$ must be equal to no less than 2750 . If $\sigma_{p} \%=15 \%$, it is necessary $N \geq 6188$ measurements to obtain the same accuracy.

\section{Conclusion}

The described researches have shown that the parameters of the experiment influence significantly the accuracy of the obtained ZIP load models. The error can vary from part to thousand of percent, depending on the range of the voltage variation $\Delta u \%$, the number of measured values $N$ and the standard deviation of load power $\sigma_{p} \%$.

The detected dependence of the static load model's error on the parameters of the experiment can be applied to solve two main problems:

- estimating the experimentally obtained static load models accuracy;

- planning the experiment for determining static load models with the prescribed accuracy.

The work was supported by Act 211 Government of the Russian Federation, contract № 02.A03.21.0006 and the Ministry of Education and Science of the Russian Federation (in the framework of state assignment, №13.1928.2014/K (project №1928)).

\section{References}

1. C.A. Baone, S. Veda, Y. Pan, W. Premerlani, J. Dai, A. Johnson, IEEE Power and Energy Society General Meeting, 2015, 7285681 (2015)

2. J.N.S. Mrudveeka, S.V.N.L. Lalitha, M. Ramamoorty, Indian J. Sci. Technol., 9 (23), 92514 (2016)

3. J. Milanović, K. Yamashita, S. Martínez Villanueva, S. Djokić, and L. Korunović, IEEE Transactions on Power Systems, 28, 3038 (2013)

4. A. Bokhari, A. Alkan, R. Dogan, M. Diaz-Aguilo, F. De Leon, D. Czarkowski, Z. Zabar, L. Birenbaum, A. Noel, R.E. Uosef, IEEE Transactions on Power Delivery, 29, 1372 (2014)

5. E. Polykarpou, E. Kyriakides, 18th Mediterranean Electrotechnical Conference, 2016, 7495363 (2016)

6. E.O. Kontis, A.I. Chrysochos, G.K. Papagiannis, T.A. Papadopoulos, IEEE Eindhoven PowerTech, 2015, 7232800 (2015)

7. X. Zhang, C. Lu, Y. Han, S. Yu, J. Wang, H. Huang, Y. Su, IEEE Power and Energy Society General Meeting, 2015, 7285620 (2015) 